\title{
PROPAGATION OF INJECTED COSMIC RAYS UNDER DISTRIBUTED REACCELERATION
}

\author{
M. Simon, W. Heinrich, K.D. Mathis \\ University of Siegen, Physics Department \\ Adolf-Reichwein-Str., 5900 Siegen, West Germany
}

\begin{abstract}
:
We here present a calculation in which we allow cosmic ray sources but also allow for reacceleration of propagating particles. It is shown that such a model can be accomodated with the data but it requires a modification of the pathlength distribution.
\end{abstract}

\section{1.) Introduction}

The cosmic ray acceleration model in which the particles gain continuously energy from stochastic processes in the interstellar space by encountering shocks leads to a logarithmic increase in the secondary-to-primary ratios with energy, which is inconsistent with observations (1). The observed decrease of the secondary-to-primary ratios may therefore suggest that a considerable part of the acceleration has taken place rapidly at the sources. Astronomical observations on the other hand indicate that shocks from supernovae do exist in the galaxy and observations in the interplanetary space show clear evidence that shock-acceleration works. Therefore we here present and discuss a concept in which we combine both ideas:

a) Cosmic rays are preaccelerated at their sources.

b) During propagation the particles are moderately reaccelerated.

\section{2.) The astrophysical assumptions}

Our model has the following astrophysical assumptions:

a) Cosmic rays are injected by discrete sources. All injected nuclei show a power law spectrum in rigidity, providing the same spectral index.

b) Particles encounter exponential age - or pathlength distribution.

c) Particles undergo nuclear collisions leading to secondary spallation products.

d) Particles, secondaries and primaries, gain rigidity when they encounter expanding supernova remnants during their propagation.

We assumed that the reacceleration is predominantly controlled by supernova remnants in a developed stage. Encounters with expanding supernova remnants in its early stage with high shock speed are very unlikely since they fill only a very small portion of the volume of the galaxy. The reacceleration by old supernova remnants on the other hand is also limited since the shock speed becomes too small to account for significant reacceleration. Thus we estimated a reacceleration around every $10^{6}$ years and allowed a 
rigidity gain of typically $10 \%$ to $30 \%$ per encounter.

\section{3.) The Monte Carlo Propagation Program}

A particle on its way can change its energy, its mean pathlength $\bar{\lambda}_{\text {es }}(E)$ and the fragmentation cross sections several times. Insfrder to describe this complex situation mathematically we developed a Monte Carlo propagation program. It is capable of following each particle through space, considering interaction, escape and reacceleration. The pathlengths, the position of interaction and the fragmentation chanels were treated as random variables with appropriate distribution functions. No ionization losses were considered so that the results are restricted to higher energies. The cross sections were derived from the semi-empirical formulae given in (2) and we used a source composition as given by (3).

\section{4.) Results}

We used the $\bar{\lambda}_{\text {esc }}(R)$-values as derived from observations by various groups under the Leaky Box approximation and adapted the following fit to these data

$$
\begin{aligned}
& \bar{\lambda}_{\text {esc }}=\mathrm{x}_{0}\left(\mathrm{~g} / \mathrm{cm}^{2}\right) \text { with } \mathrm{x}_{0}=8.0 \text { for } \mathrm{R} \leqq 6(\mathrm{GV}) \\
& \bar{\lambda}_{\text {esc }}=\mathrm{x}_{0}(\mathrm{R} / 6)^{-\alpha} \text { with } \mathrm{x}_{0}=8.0 \text { and } \alpha=0.5
\end{aligned}
$$

and allowed for reacceleration.

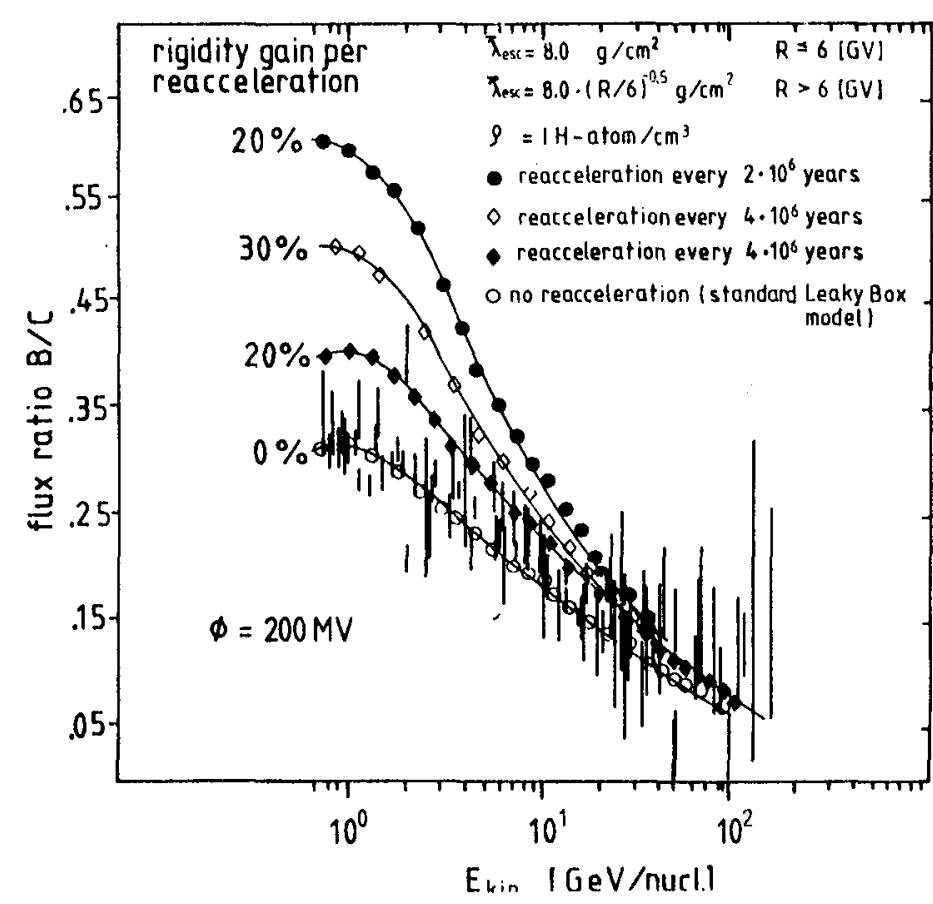

Fig.1: The calculated $\mathrm{B} / \mathrm{C}-\mathrm{flux}$ ratio under different reacceleration conditions.
The result is shown in Fig.1. The Leaky Box approximation is in good agreement with the data. However by allowing for reacceleration the calculated B/Cflux ratio increases considerably and falls well above the experimental data. The ratio at lower energies is more effected than at higher energies. High energy particles stay too short in the galaxy to be effectively reaccelerated. There are different reasons for the increase in the secondary-to-primary ratio. 
1) The energy dependence of the fragmentation cross sections, (4). More secondaries at low energies can be produced which then after reacceleration show up at higher energies. But this effect does not necessarily lead to an enhanced production of secondaries because the cross sections do not always increase with decreasing energy.

2) Reacceleration always shovels particles of lower energies to higher energies and the longer a particle stays in the galaxy the higher is the probability to obtain more energy. There are only a few particles with long residence times since the age-distribution decreases exponentially. But these few particles, which contribute after reacceleration to higher energies, have to be weighted by the steep falling injection spectrum. Particles which have encountered a number of reaccelerations are those out of the tail of the exponential pathlength distribution. A large amount of traversed matter translates to more secondaries. This effect always results in an enhanced secondary-to-primary ratio.

\section{5.) Discussions}

The more effective production of secondaries can be compensated by reducing the mean traversed matter. Results of such a calculation are shown in Fig. 2. The propagation parameters

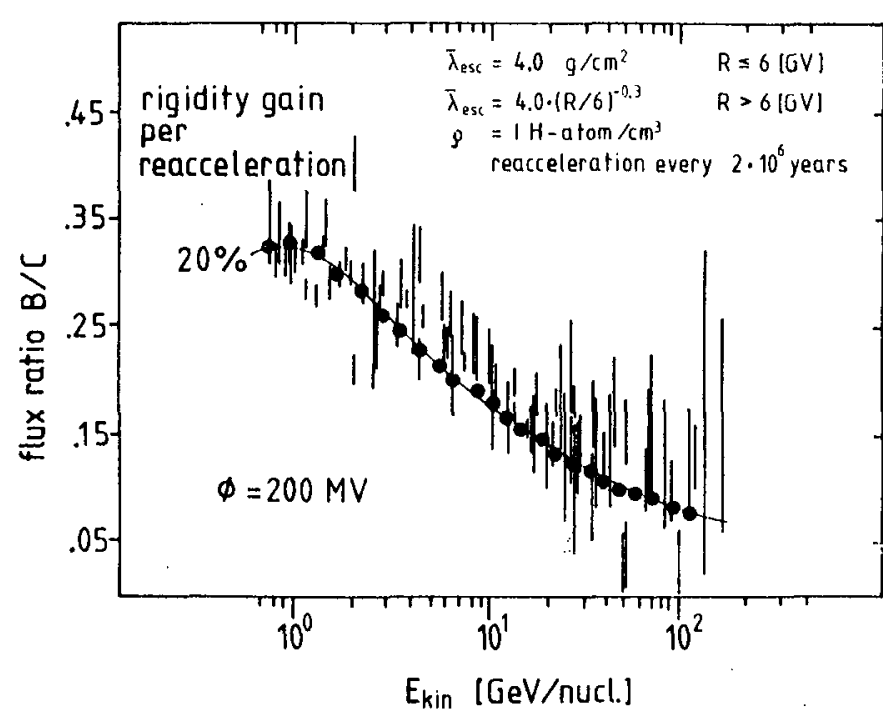

Fig.2: The B/C-flux ratio under reacceleration conditions, calculated with a modified pathlength distribution. are given in the

figure. Under these conditions we found that the mean traversed matter around $1 \mathrm{GeV} / \mathrm{n}$ is closer to $4 \mathrm{~g} / \mathrm{cm}^{2}$ instead of $8 \mathrm{~g} / \mathrm{cm}^{2}$ and the energy dependence of $\bar{\lambda}_{\text {esc }}$ is more 1 ike $\bar{\lambda}_{\text {esc }} \sim R^{-0,3}$ instead
of $\lambda_{\text {esc }} \sim R^{-0,5}$. The calculations show the following trends. The stronger the reacceleration is (more frequent and/or higher rigidity gain) the less traversed matter with an even flatter energy dependence is required in order to fit the data. The weaker the reaccelera-

tion is the closer it reflects the standard Leaky Box situation. Measurements on the energy dependent decrease of the secondary-to-primary ratios beyond $100 \mathrm{GeV} / \mathrm{n}$ may place limits on the reacceleration conditions if one assumes that the weaker energy dependence of $\bar{\lambda}_{\text {esc }}(E)$ extends to higher energies, Fig. 3. 


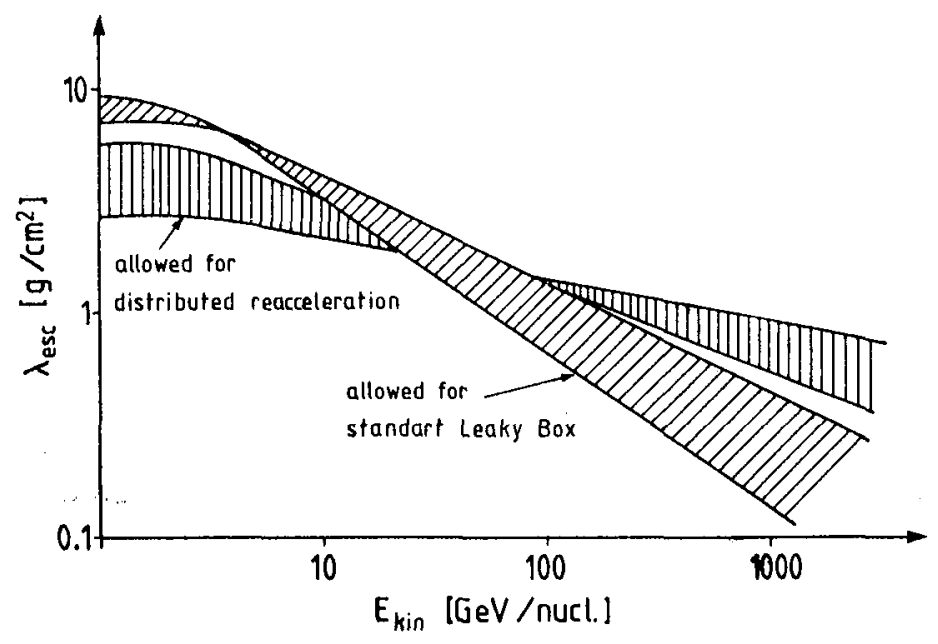

Fig.3: Schematic drawing of the energy dependence of $\lambda_{\text {f }}$ ( $E$ ) as predicted by the standard Leaky Box and the distributed reacceleration model.

\section{6.) Conclusions}

Reacceleration has also an impact on the surviving fraction of radioactive cosmic ray nuclei. In the Leaky Box model the surviving fraction is solely a function of the interstellar gas density. By allowing for distributed reacceleration the mean Lorentz factor is less than that determined by the arriving energy. This leads to a more frequent decay and causes an underestimation of the gas density by interpreting the data in the framework of the Leaky Box model. flux ratio are available around $100 \mathrm{MeV} / \mathrm{n}$ but this is an energy range where we are not capable of making meaningful calculations since our computer program at the present state does not take ionization losses into account. But in general, reacceleration works towards a shorter cosmic ray age due to less amount of traversed matter and a smaller mean Lorentz factor. When reacceleration is allowed we are probably closer to $10^{6}$ years than to $10^{7}$ years. The flux ratios in the energy regime around $100 \mathrm{MeV} / \mathrm{h}$ may be very much affected by cross section variations which occur below $100 \mathrm{MeV} / \mathrm{n}$. It is observed that some cross sections show resonance-like increases below $100 \mathrm{MeV} / \mathrm{n}$. B10 and B11production for instance from the p-016-interaction is 4 times higher around $60 \mathrm{MeV} / \mathrm{n}$ than around $200 \mathrm{MeV} / \mathrm{n}$. Under reacceleration condition the high production rate of these particles should influence the result also above $100 \mathrm{MeV} / \mathrm{n}$. The effect may be somewhat washed out by ionization losses but measurements in this low energy regime should open new possibilities to check on the validity of reacceleration processes.

\section{References}

(1) Cowsik, R. 1980, Ap.J. 241, 1195

(2) Silberberg, R., Tsao, C.H. 1973, Ap.J. 25,315 and 15th ICRC, Plovdiv 2, 89, 1977

(3) Cameron, A.G.W. 1973, Space Sci.Rev. 15, 121

(4) Silberberg et al. 1983, Phys.Rev.Lett. 51,1217 